\title{
SAÚDE COLETIVA E ATIVIDADE FÍSICA: RECORTES ATUAIS DE SUA ATUAÇÃO
}

Aguinaldo Gonçalves

Ana Claudia da Silva Moura

Ana Paula Martins Vicentin

Carla Cristina Cuoco Léo

Fabrício Boscolo Del Vecchio

Glauca Gonçalves Mantellini

Gláucia Cristina de Castro

Sérgio Ricardo Pasetti

\section{Resumo}

O Grupo de Saúde Coletiva/Epidemiologia e Atividade Física da Unicamp apresenta recortes atuais de sua atuação no desenvolvimento de projetos técnicos a partir de três núcleos temáticos de interesse: i) caracterizações de metodologias de pesquisa e ensino empregadas em seu interior; ii) exploração de relações entre níveis de Qualidade de Vida e diferentes programas de Atividade Física e iii) aspectos do binômio corpo-movimento em ações de controle da hanseníase. Especificamente, apresentam-se como resultados expressivos os significados que podem ser explicitados pela análise conjunta de diferentes projetos conduzidos de forma integrada por mesma equipe acadêmica.

\section{Palavras-Chave:}

Educação superior; Educação Física e treinamento; Hanseníase; Saúde pública; Saúde coletiva; Qualidade de vida.

\section{COLLECTIVE HEALTH AND PHYSICAL ACTIVITY: CURRENT CLIPPINGS OF THEIR PERFORMANCE}

\begin{abstract}
The Group of Public Health/Epidemiology and Physical Activity from the State University of Campinas displays here segment of its nowday experience on developing teaching and research projects. These studies are organised in three nuclei: i) characterization of applied technical methodologies; ii) exploitation of relationships between Quality of Life levels and different programmes of Physical Activity and iii) aspects of body-movement in actions of leprosy control. It is considered, as outstanding meaning of obtained results, the possibility of integrated analysis of distinct projects conducted by the same scientif team.
\end{abstract}

\section{Key-Words:}

Higher education; Leprosy; Physical Education and training; Public health; Quality of Life. 


\section{Introdução}

Entre os numerosos aspectos que destacam o Grupo de Saúde Coletiva e Atividade Física da Unicamp no conjunto de realizações acadêmicas em nosso meio, estão a diversidade, profundidade e abrangência dos projetos realizados, desde respectiva criação há quase vinte anos. A última atualização de sua produção, ocorrida há aproximadamente dois meses (www.unicamp.br/fef), registra a publicação de 386 textos em periódicos correntes e em anais de congressos, distribuídos em cinco principais linhas de pesquisa, a saber: i) Grupos Populacionais (GP); ii) Saúde Coletiva e Atividade Física (SCAF); iii) Estudos Colaborativos Multicêntricos (ECM); iv) Pesquisa e Informação em Ciência do Esporte (PICE); v) Epidemiologia das Lesões Desportivas (ELD).

O objetivo da presente comunicação consiste em descrever e apreciar, de forma indicativa, os principais projetos que se desenvolvem atualmente em três das linhas mencionadas (PICE; GP e ECM). Trata-se, respectivamente de: i) caracterizações de metodologias de pesquisa e ensino adotadas no interior do Grupo; ii) relações entre níveis de Qualidade de Vida e diferentes programas de Atividade Física (AF) e iii) aspectos do binômio corpo-movimento em ações de controle da endemia hansênica a níveis local e internacional.

\section{Caracterizações de Metodologias de Pesquisa e Ensino Adotadas no Interior do Grupo}

Em busca de conhecer as modalidades metodológicas que temos adotado, investigaram-se nossas publicações em periódicos e anais, alocando-as em duas grandes categorias, quantitativa e qualitativa (MINAYO, SANCHES, 1993). Posteriormente, identificaram-se subgrupos que especificam a abordagem utilizada em cada estudo (PADOVANI, 2001), segundo as cinco Linhas de Pesquisa já mencionadas.

Observamos que, em geral, a metodologia mais empregada foi a quantitativa, e nesta, os testes estatísticos inferenciais. Corroboram neste sentido GP, ECM e ELD, com destaque para o teste paramétrico univariado, a distribuição de freqüência e análise não paramétrica, respectivamente. Já em SCAF e PICE, a predominância foi da qualitativa, com textos de revisão e relatos de experiência, como mais freqüentes. Portanto, as linhas empiricistas demonstram empregar mais procedimentos quantitativos analíticos e as doutrinárias, qualitativos.

À atuação em pesquisa também interessa conhecer de forma sistemática o ensino oferecido nos diversos cursos que mantemos em extensão, graduação e pós senso estrito e senso lato. Na graduação, o Grupo produz conhecimento singular na área, sendo o responsável pela introdução da Saúde Coletiva (SC) no 
currículo da Faculdade de Educação Física/Unicamp, em 1997, realização pedagógica pioneira a nível nacional, com a criação e oferta das disciplinas anuais: "Saúde Coletiva e Atividade Física" e "Saúde Coletiva e Performance Humana” (UNICAMP, 2006).

Destas, a segunda aprofunda os temas voltados à área de Saúde Coletiva/Epidemiologia com enfoque no campo da AF em grupos populacionais específicos, sendo objeto de interesse de um de nossos mestrados (MOURA, 2006). Baseando-se no trabalho conjunto do Grupo na construção e operacionalização do plano de curso até a avaliação discente, tratou-se de pesquisa quali-quantitativa formulada a fim de descrever e analisar uma de suas edições anuais nos períodos diurno e noturno.

A execução do curso consistiu de quatro blocos: i) aplicação de bateria de vinte testes de múltipla escolha para verificação e esclarecimento das informações prévias dos alunos; ii) apresentação discente em grupos sobre sites on-line de instituições representativas da área; iii) exposição de Bibliografia Básica, previamente selecionada, sobre fundamentos, evolução, controvérsias e aplicações da intervenção profissional na área e iv) discussão realizada por integrantes do GSCEAF relacionando as valências físicas resistência, força, flexibilidade, velocidade e coordenação motora e seus aspectos epidemiológicos (GONÇALVES et al., 2004).

De modo geral, os resultados apreendidos evidenciaram ausência de contato e pouco interesse prévios dos alunos com os conceitos e práticas de Saúde Coletiva e Educação Física, em decorrência à inclusão restrita até então de temas relacionados a Saúde no curso e ausência de estágio supervisionado de campo. Em conseqüência, entre as conclusões destacam-se aspectos da Saúde Coletiva e AF como campo a ser inteirado pelos profissionais da Educação Física e ampliação da acessibilidade aos conhecimentos específicos com a utilização da internet na Disciplina.

\section{Relações Entre Níveis de Qualidade de Vida e Atividade Física em Diferentes Grupos Populacionais}

Segmento de pesquisa que tem tido destaque, no âmbito da SC e Educação Física, constitui-se na Qualidade de Vida (QV) das populações.

Ela diz respeito ao cotidiano das pessoas, envolvendo saúde, transporte, educação, moradia, trabalho e participações nas decisões que lhes dizem respeito. Ao usar o termo QV, pode-se estar indicando, entre outros, bem estar pessoal, posse de bens, participações em deliberações coletivas, ou seja, é aplicado com inúmeros sentidos. (GONÇALVES, VILARTA, 2004a, p. 3). 
Dentre as considerações mais utilizadas para sua definição, a Organização Mundial da Saúde (OMS) explicita tratar-se da visão do indivíduo de sua posição na vida, no contexto da cultura e do sistema de valores em que vive e em relação aos seus objetivos, expectativas, padrões e preocupações (WHO, 1995). Conceito cunhado no interior da FEF/Unicamp diz respeito à percepção subjetiva da participação no processo de produção, circulação e consumo de bens (GONÇALVES, 2004). A partir dessa abordagem registram-se duas concepções relevantes para compreender QV: uma, referente a dimensões emocionais, culturais e sociais, e outra, objetiva, acerca das condições materiais, posição da pessoa na vida e relações estabelecidas na sociedade (GONÇALVES; VILARTA, 2004b).

Na perspectiva biomédica, tem-se a expressão Qualidade de Vida Relacionada à Saúde (QVRS), constructo multidimensional que contempla as singularidades das pessoas, segundo variáveis de interesse (SNEEUW, SPRANGERS, AARONSON, 2002). Para medir QVRS existem mais de mil instrumentos específicos (PASCHOAL, 2001). Dentre os mais empregados, destacam-se o WHOQOL-Abreviado, desenvolvido pela OMS (WHO, 1998) e o Medical Outcome Study Short Form-36, SF-36 (WARE et al., 1998). Com o cuidado de ponderar facetas sócio-culturais e econômicas, elementos advindos deles podem ser interpretadas como referências dos grupos populacionais nos quais são aplicados, auxiliando nos respectivos conhecimentos e manejo do processo saúde-doença-cuidado.

No interior do GSCEAF ambos os questionários têm sido adotados. Em estudo de Vicentin et al. (2006), em região adstrita à Universidade Estadual de Campinas, tomaram-se os valores de mulheres de 30 a 55 anos, residentes na Região do São Marcos, Campinas, SP. Pasetti et al. (2004a) investigaram voluntárias de 38 a 57 anos, na prática do Deep Water Running (DWR). Na perspectiva da Saúde do Trabalhador, Del Vecchio et al. (2006) mensuraram os níveis de QV das pessoas intoxicadas cronicamente por mercúrio, no meio urbano industrial.

Vale lembrar que, mesmo fragmentários, os recortes decorrentes das três investigações apresentam situações reveladoras. Dessa forma, na relativização dos escores absolutos, em escala padronizada de zero a cem, tentou-se correspondência entre os diferentes domínios abordados pelo WHOQOL-Abreviado e pelo SF-36, em comparações expressas na tabela 1, identificando-se que o componente físico do WHOQOL-Abreviado revela percepções próximas ao funcionamento físico e dor corporal do SF-36; o psicológico tem proximidades com função emocional e saúde mental. Nos aspectos extrínsecos, o social 
guarda relação com a função física e funcionamento social; por fim, o meio ambiente compõe-se com vitalidade e função emocional.

Observa-se, nos dois primeiros estudos apresentados, que, apesar de as faixas etárias serem próximas, os escores de QV para o primeiro são superiores em todos os domínios, com exceção do psicológico. As características de ambos os grupos podem explicar este fato: as mulheres do primeiro, obesas, tinham em sua maioria acesso a emprego, automóvel e residência própria; já o segundo foi composto por moradoras de uma das regiões mais carente da cidade, com alto índice de tráfico de entorpecentes e mortes por causas externas, parte das casas sem energia elétrica e o saneamento básico atendendo a segmento muito limitado do bairro. Ou seja, entendeu-se a resposta coerente com a materialidade de vida a que estão expostas, inobstante participaram ambos os contingentes dos treinamentos atléticos assemelhados que lhes foram aplicados.

Tabela 1- Medianas dos valores percentuais dos domínios de QV dos diferentes grupos mencionados

\begin{tabular}{|c|c|c|c|c|c|c|c|c|}
\hline \multirow{2}{*}{$\begin{array}{c}\text { Estudo } \\
\text { (População e Idade em anos) }\end{array}$} & \multicolumn{8}{|c|}{ Domínios } \\
\hline & & & Psic & gico & & cial & Meio & bbiente \\
\hline $\begin{array}{l}\text { Vicentin et al., 2006 } \\
\text { (Sedentárias, 30-55) }\end{array}$ & \multicolumn{2}{|c|}{57,14} & \multicolumn{2}{|c|}{58,33} & \multicolumn{2}{|c|}{66,67} & \multicolumn{2}{|c|}{46,88} \\
\hline $\begin{array}{l}\text { Pasetti, 2005 } \\
\text { (Obesas, 38-57) }\end{array}$ & \multicolumn{2}{|c|}{67,86} & \multicolumn{2}{|c|}{58,33} & \multicolumn{2}{|c|}{75,00} & \multicolumn{2}{|c|}{56,25} \\
\hline \multirow{2}{*}{$\begin{array}{l}\text { Del Vecchio, } 2005 \\
\text { (Intoxicados por } \mathrm{Hg}, 18-52 \text { ) }\end{array}$} & $\mathrm{PF}$ & BP & $\mathrm{RE}$ & $\mathrm{MH}$ & $\mathrm{RP}$ & SF & GH & VI \\
\hline & 40,00 & 22,20 & 0,00 & 36,00 & 0,00 & 50,00 & 30,00 & 30,00 \\
\hline
\end{tabular}

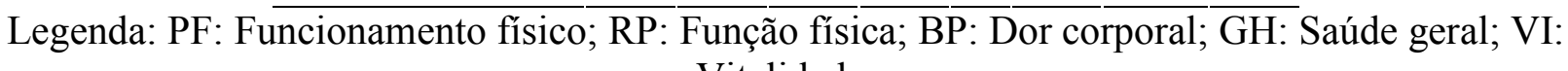
Vitalidade;

SF: Funcionamento social; RE: Função emocional; MH: Saúde mental.

Já aqueles que sofreram exposição ao mercúrio, que por sua vez conduz a prejuízos neuropsiquiátricos, danos pulmonares e renais, relatam percepção baixa de QV. Quando se contemplam os valores das três investigações, as pessoas acometidas pelo metal apresentam cifras expressivamente inferiores às verificadas entre aquelas. Excluindo o funcionamento social, com $50 \%$, os doentes relatam escores entre zero e $40 \%$. As funções física e emocional, em consonância com os domínios psicológico e social do WHOQOL, reforçam a circunstância de prejuízos gerados pelo agravo crônico. Além disso, ambas dizem respeito às limitações decorrentes de problemas físicos e emocionais, principalmente quanto às tarefas e atividades da vida diária e no trabalho, assim como no âmbito social. 
Com efeito, autores indicam, tanto na realidade nacional (CONTE, GONÇALVES, 2004), como internacional (KELL, BELL, QUINNEY, 2001), as potencialidades da AF regular na percepção subjetiva de QV. Nesse sentido, ampliando as observações da tabela 1, aplicaram-se conceitos da Educação Física e Treinamento Desportivo, em práticas de 13 semanas de hidroginástica (VICENTIN et al., 2006) e 17, de DWR (PASETTI et al., 2004b), que pudessem gerar eventuais benefícios da AF na QV das pessoas (Tabela 2). De pronto, nota-se nos dois estudos evolução dos respectivos valores.

Tabela 2 - Comparação dos valores dos efeitos das intervenções de DWR e hidroginástica na QV dos respectivos grupos

\begin{tabular}{|c|c|c|c|c|c|c|c|c|}
\hline \multirow{3}{*}{ Estudo } & \multicolumn{8}{|c|}{ Domínios } \\
\hline & \multicolumn{2}{|c|}{ Físico } & \multicolumn{2}{|c|}{ Psicológico } & \multicolumn{2}{|c|}{ Relações Sociais } & \multicolumn{2}{|c|}{ Meio Ambiente } \\
\hline & $\mathrm{AI}$ & $\mathrm{AF}$ & $\mathrm{AI}$ & $\mathrm{AF}$ & AI & $\mathrm{AF}$ & AI & $\mathrm{AF}$ \\
\hline Pasetti (2005) & 67,86 & $82,14^{*}$ & 58,33 & $70,83^{*}$ & 75,00 & $75,00^{*}$ & 56,25 & 62,50 \\
\hline Vicentin et al. (2006) & 57,14 & $64,29 *$ & 58,33 & $64,58^{*}$ & 66,67 & 66,67 & 46,88 & $54,69 *$ \\
\hline
\end{tabular}

AI: Avaliação inicial; AF: Avaliação final;

*: Diferenças significantes para avaliações iniciais e finais $(p>0,05)$.

Diante dos achados referidos, pontua-se que atividades físicas diferenciadas podem contribuir para a evolução da QV. No domínio físico nota-se melhora significante dos resultados tanto na DWR quanto na hidroginástica. $\mathrm{O}$ mesmo ocorreu para o indicador psicológico: a prática de exercícios contribuiu para a evolução favorável de dados emocionais como humor, auto-estima e imagem corporal, podendo a menor exposição do corpo em atividades aquáticas e alterações da composição corporal ter favorecido os resultados (BECKER, 2000).

Para o domínio relações sociais, ressalta-se a primeira divergência dos estudos com atividades aquáticas. Pasetti et al. (2004b) encontraram evolução significativa, talvez porque a socialização de pessoas com características semelhantes tenha favorecido troca de experiências, relatos e facilitação do relacionamento social (CONTE; GONÇALVES; 2004), contrariamente à intervenção com hidroginástica. Neste sentido, registra-se aí a composição por três perguntas que abordam unicamente as relações pessoais, vida sexual e apoio de amigos, e aponta, conforme já relatado em outras oportunidades (VICENTIN et al., 2004; 2006), para entendimento restrito de conceito bastante amplo.

$\mathrm{Na}$ análise do meio-ambiente, percebe-se que apesar de não ocorrerem, de fato, mudanças materiais, como por exemplo, maior nível de segurança e condições dignas de moradia, o grupo caminhou para 
outra leitura da realidade cotidiana; esse domínio expressa claramente facetas como oportunidades de recreação e lazer, educação, moradia, ambiente físico e satisfação no trabalho, indicando para a representatividade que foi dada pelas mulheres da comunidade carente à participação em programa de AF no meio aquático. Coerentemente, o trabalho com DWR não expressou efeito significativo nesse contexto.

\section{Aspectos do Binômio Corpo-movimento em Ações de Controle da Endemia Hansênica a Níveis Local e Internacional}

Entre as aplicações do GSCEAF, a Hanseníase tem se apresentado como objeto de análise dos perfis epidemiológicos das doenças transmissíveis e suas relações com componentes bio-sociais, entre os quais se encontra a atividade física. Dos projetos desenvolvidos sobre esse tema, destacamos os dois mais recentes.

No primeiro (MANTELLINI, 2006), as incapacidades físicas da hanseníase (IFH) foram apreendidas como interesse central, no sentido de se discutir e deslindar se, na atualidade, constituem coisa do passado ou problema do futuro, a partir dos programas específicos de controle. A reconstrução metodológica adotada foi de natureza qualitativa, partindo-se de textos acadêmicos e de documentos de organismos nacionais e internacionais voltados à epidemiologia da hanseníase: fulcrada nas técnicas de revisão bibliográfica e análise de conteúdo, situou-se como de tipificação documental categorial freqüêncial contigencial (BARDIN, 2004), incorporando 252 textos técnico-científicos, catalogados a partir do instrumento de sistematização Reference Manager ${ }^{\circledR} 11$.

Uma das principais tendências das IFH, repetidamente afloradas neste contexto, foi o reconhecimento oficial da importância que vêm merecendo, em flagrante contraponto com a prioridade que lhes é conferida (WHO, 2005). Objetivamente, releva-se que as decisões, investimentos e práticas, decorrentes das políticas recentes referentes à hanseníase, têm centralizado seus saberes e agires na questão da ação bacilar direta dentro e fora do organismo humano. Em conseqüência, abriu-se largo espaço para atuações de organizações não governamentais e associações congêneres quanto aos aspectos reabilitacionais da moléstia (KALK, 2003). Tal incursão pelo plano macro-analítico do passado se complementa com a discussão da intervenção pela Educação Física e com o cuidado com as IFH a partir da Saúde Coletiva em revisita crítica que se procede a dimensões mais estritamente aplicadas das IFH com questões contemporâneas do corpo e do movimento inseridas nas várias interfaces da doença e nas alterações 
corporais individuais: algumas atividades adaptadas a esta realidade - treinamento sensorial e percepção cinestésica, jogos de simples organização, dança e movimentos rítmicos, alguns esportes coletivos e alongamento muscular - são indicadas como componentes da pós-reabilitação e reinserção social (GONÇALVES, 2001).

A decorrência desta realidade é tomada, a seguir, na direção de respectivas conclusões: projeta-se que a expansão e crescimento da incidência da doença colocam reptos econômicos e sanitários a desafiar desde o modelo neoliberal de organização societária mundial até competências específicas das ações das equipes de saúde em campo, incluindo os profissionais de Educação Física (BAUMAN, 1999).

E nesse sentindo do adaptar-se através e para o corpo junto a populações específicas é que se posiciona o segundo projeto. Constituiu-se com prática local, na cidade de Campinas, SP, verificando a contribuição do Teatro Interativo da Sorri Sorocaba, no diagnóstico precoce de hanseníase, através de parceria entre o sistema público de saúde e organizações não governamentais. Tal manifestação artística constitui adaptação da técnica japonesa chamada bunraku, que consiste na atuação de manipuladores como sombras de bonecos, vestindo trajes pretos, inclusive capuz (CASTRO, 2005). Para tanto, a pesquisa foi alicerçada nas bases da teoria do interacionismo simbólico, segundo a qual os indivíduos são ativos e aprendem a dar significado às coisas através da interação social, sendo passível de mudanças no decorrer do processo interpretativo (CHARON, 1989).

A iniciativa decorreu de colaboração entre o GSCEAF, Sorri/Sorocaba, Centro de Saúde Municipal Santa Mônica e o Centro Assistencial Vedruna, esta uma organização religiosa com atuação local (VEDRUNA, 2002). A área de aplicação é constituída pela já referida Região do São Marcos, Zona Norte de Campinas, SP. Foram realizadas seis apresentações com o tema referente à hanseníase, para 120 crianças e adolescentes, com idade entre 7 e 14 anos e 30 mães, todos participantes de atividades no Centro Assistencial Vedruna. Resultados exploratórios indicam que as intervenções decorrentes do projeto, assim como os protagonistas envolvidos no processo, não foram suficientes para modificar a sistemática das limitadas instituições correspondentes, sobretudo as de compromisso predominantemente assistencial, pois se expressam como barreiras importantes à ação integrada dos serviços. 


\section{Referências Bibliográficas}

BARDIN, L. Análise de conteúdo. Lisboa: Edições 70, 2004. 223 p.

BAUMAN, Z. Globalização: as conseqüências humanas. Rio de Janeiro: Jorge Zahar, 1999. 145 p.

BECKER, B. E. Princípios físicos da água. In: RUOTI, R. G.; MORRIS, D. M.;COLE, A. (Org.). Reabilitação aquática. São Paulo: Manole, 2000. p. 17-28.

CASTRO, G. C. Transformação social por meio do movimento Sorri: do isolamento à inclusão. In: GONÇAlVES, A.; GUTIERREZ, G. L.; VILARTA, R. (Org.). Gestão da qualidade de vida na empresa. Campinas, SP: IPES, 2005.

CHARON, M. Symbolic interacionism: an introduction, an interpretation, an integration. California: Prentice-Hall, 1989.

CONTE, M.; GONÇALVES, A. Qualidade de vida e aptidão física. In: GONÇALVES, A.; VILARTA, R. (Org.). Qualidade de vida e atividade física: explorando teoria e prática. São Paulo: Manole, 2004. p. 257-287.

DEL VECCHIO, F. B. Qualidade de vida e atividade física em intoxicados por mercúrio: estudo observacional transversal descritivo. 2005. 97f. Dissertação (Mestrado em Ciências do Esporte) Faculdade de Educação Física, Universidade Estadual de Campinas, Campinas, 2005.

DEL VECCHIO, F. B. et al. Evaluating quality of life in mercury intoxicated people in urban-industrial environments through short-form 36 application. Revista Brasileira de Medicina, 2006. (no prelo).

GONÇALVES, A. Apresentação. In: GONÇALVES, A. VILARTA, R. (Org.). Qualidade de vida e atividade física: explorando teoria e prática. São Paulo: Manole, 2004. p. XIV.

. et al. Educação Física e a produção do conhecimento: o caso da saúde coletiva e performance humana. In: CONGRESSO LATINO-AMERICANO DE EDUCAÇÃO FÍSICA DA UNIMEP, 3., 2004, Piracicaba-SP. Anais... Piracicaba: UNIMEP, 9 a 12 jun. 2004. 1 CD-ROM.

GONÇALVES, A.; VILARTA, R. Qualidade de vida: identidades e indicadores. In: . (Org.).

Qualidade de vida e atividade física: explorando teoria e prática. São Paulo: Manole, 2004a. p. 3

. Qualidade de vida: concepções básicas voltadas à saúde. In:

. (Org.). Qualidade de vida e atividade física: explorando teoria e prática. São Paulo: Manole, 2004b. p. 27-62.

GONÇALVES, G. Caminhando na cidadania para além das incapacidades físicas em hanseníase: atividade física a partir de unidade de referência do Sistema Único de Saúde. 2001. 85f. Dissertação. (Mestrado em Educação Física) - Faculdade de Educação Física, Universidade Estadual de Campinas, Campinas, 2001. 
KELL, R. T.; BELL, G.; QUINNEY, A. Musculoskeletal fitness, health outcomes and quality of life. Sports Medicine, v. 31, n. 1, p. 863-873, 2001.

KALK, A. A cooperação entre uma ONG e os estados "anfitriões" no controle da hanseníase na América Latina. Cadernos de Saúde Pública, v. 19, n. 2, p. 663-666, 2003.

MANTELLINI, G. G. Incapacidades físicas em hanseniase e atividade fisica : coisa do passado ou problema do futuro?. 2005. 135 f. Tese de (Doutorado em Ciências do Esporte) - Faculdade de Educação Física, Universidade Estadual de Campinas, Campinas, 2006.

MINAYO, M.S.C.; SANCHES,O. Qualitativo-quantitativo: oposição ou complementaridade? Cadernos de Saúde Pública, Rio de Janeiro, v. 9, n. 3, p. 239-262, 1993.

MOURA, A. C. S. MH 607 FEF/UNICAMP, onde Saúde coletiva e performance humana se encontram: uma pesquisa-ação. 2006. 101f. Dissertação (Mestrado em Educação Física) - Faculdade de Educação Física, Universidade Estadual de Campinas, Campinas, 2006.

PADOVANI, C. R. Noções Básicas de bioestatística. In: CAMPANA, A. O. et al. Investigação científica na área médica. São Paulo: Manole, 2001. p. 153-186.

PASCHOAL, S. M. P. Qualidade de vida do idoso: elaboração de um instrumento que privilegia sua opinião 2001. 263 f. Dissertação (Mestrado em Medicina Preventiva) - Faculdade de Medicina, Universidade de São Paulo , 2001. p. 70.

PASETTI, S. R., GONÇALVES, A., PADOVANI, C. R. Deep water running para Redução da Gordura Corporal. In: CONGRESSO CIENTÍFICO LATINO AMERICANO DE EDUCAÇÃO FÍSICA E ESPORTES DA UNIMEP, 3., 2004, Piracicaba, Anais... Piracicaba: UNIMEP, 9 a 12 jun 2004a. 1 CDROM.

- Corrida em água profunda para melhora da aptidão física de mulheres obesas: estudo experimental de grupo único. In: SIMPÓSIO INTERNACIONAL DE CIÊNCIAS DO ESPORTE, 27., 2004, São Paulo. Anais... São Paulo: CELAFISCS, p.197, 7 a 9 out 2004b.

PASETTI, S. R. Deep water running para redução da gordura corporal em mulheres na meia idade: estudo de intervenção em Campinas-SP. 2005. 125 f. Dissertação (Mestrado em Ciências do Esporte) Faculdade de Educação Física, Universidade Estadual de Campinas, Campinas, 2005.

SNEEUW, K. C. A.; SPRANGERS, M. A. G.; AARONSON, N. K. The role of health care providers and significant others in evaluating the quality of life of patients with chronic disease. Journal of Clinical Epidemiology, v. 55, n. 6, p. 1130-1143, 2002. 
UNICAMP. Universidade Estadual de Campinas. Pró-Reitoria de Graduação. Catálogo de Graduação 2006. Disponível em: <http://www.unicamp.br/prg/dac>. Acesso em: 05 mai. 2006.

VEDRUNA, Associação Civil Carmelitas da Caridade. Centro assistencial Vedruna: defendendo e promovendo a vida. Campinas: São Paulo, 2002. (Folheto Informativo.)

VICENTIN, A. P. M., et al. Efeitos de programa quasi-experimental de hidroginástica na qualidade de vida de mulheres sedentárias aponta para consideração do domínio social no WHOQOL-Bref. In: JORNADA SUL-RIO-GRANDENSE DE PSIQUIATRIA DINÂMICA, 22. ENCONTRO IBEROAMERICANO DE QUALIDADE DE VIDA, 2. 2004. Porto Alegre, Anais... Porto Alegre: Centro de Eventos da AMRIGS, 19 a 21 ago. 2004.

VICENTIN, A. P. M., PADOVANI, C. R., GONÇALVES, A. Qualidade de vida, empowerment e atividade física em mulheres brasileiras o caso da hidroginástica no Jardim São Marcos, Campinas-SP. In: VILARTA, R. et al. (Org). Qualidade de Vida e Fadiga Institucional. Campinas: IPES Editorial, 2006.

WARE, J. E. et al. The factor structure of the SF-36 health survey in 10 countries: Results from the IQOLA project. Journal of Clinical Epidemiology, v. 51, n. 11, p. 1159-1165, 1998.

WHO. World Health Organization. The World Health Organization Qualitity of Life Assessment (WHOQOL): position paper from the World Health Organization. Social Science Medicine, v. 41, n. 10 , p. 1403-1409, 1995.

WHO. The WHOQOL Group. Development of the World Health Organization WHOQOL-BREF Quality of Life Assesment. Psychological Medicine, v. 28, n. 3, p. 551-558. 1998.

WHO. Global leprosy situation, 2005. Weekly Epidemiological Record, v. 80, n. 34, p. 289-295, 2005. 
Aguinaldo Gonçalves

Faculdade de Educação Física/UNICAMP

Ana Claudia da Silva Moura

Faculdade de Educação Física/UNICAMP

Ana Paula Martins Vicentin

Faculdade de Educação Física/UNICAMP

Carla Cristina Cuoco Léo

Faculdade de Educação Física/UNICAMP

Fabrício Boscolo Del Vecchio

Faculdade de Educação Física/UNICAMP

Glauca Gonçalves Mantellini

Faculdade de Educação Física/UNICAMP

Gláucia Cristina de Castro

Faculdade de Educação Física/UNICAMP

Sérgio Ricardo Pasetti

Faculdade de Educação Física/UNICAMP 